\title{
Análise do Problema de Advecção e Difusão Bimodal Unidimensional
}

\author{
JADER LUGON JUNIOR ${ }^{1 *}$, PEDRO PAULO GOMES WATTS RODRIGUES ${ }^{2}$, \\ LUIZ BEVILACQUA ${ }^{3}$, GISELE MORAES MARINHO ${ }^{4}$, DIEGO CAMPOS KNUPP ${ }^{5}$, \\ JOÃO FLÁVIO VIEIRA VASCONCELLOS ${ }^{6}$ e ANTÔNIO J. SILVA NETO ${ }^{7}$
}

Recebido em 10 de dezembro de 2018 / Aceito em 18 de fevereiro de 2020

\begin{abstract}
RESUMO. Este artigo apresenta a metodologia para a solução de um problema de advecção e difusão bimodal unidimensional utilizando o Método de Diferenças Finitas. Além do termo de transporte advectivo e da difusão primária (que corresponde ao fluxo de Fick), a equação da difusão bimodal inclui um termo relativo à um fluxo secundário que é modelado por um termo diferencial de quarta ordem. O problema foi analisado para diferentes condições iniciais e de contorno, sendo os resultados compatíveis com os apresentados em trabalhos anteriores da literatura.
\end{abstract}

Palavras-chave: difusão bimodal, difusão anômala, Método de Diferenças Finitas, equação diferencial de quarta ordem.

\section{INTRODUÇÃO}

O modelo universalmente adotado para descrever a difusão de um soluto em um meio contínuo baseia-se na lei de Fick. Contudo, há na literatura diversos relatos que descrevem processos difusivos que não seguem esta lei $[5,8,9,10,13,15]$.

Bevilacqua e coautores $[1,2,3]$ apresentaram uma formulação analítica para descrever o processo difusivo em que ocorre uma forma de retenção do fluxo de Fick em um meio rea-

\footnotetext{
*Autor correspondente: Jader Lugon Junior - E-mail: jljunior@iff.edu.br

${ }^{1}$ Programa de Pós Graduação em Engenharia Ambiental, IFF, Macaé, RJ, Brasil - E-mail: jljunior@iff.edu.br http://orcid.org/0000-0001-8030-0713

2 DMC, IPRJ/UERJ, Nova Friburgo, RJ, Brasil - E-mail: pwatts@iprj.uerj.br http://orcid.org/0000-0002-9602-9785

${ }^{3}$ COPPE/UFRJ, Rio de Janeiro, RJ, Brasil - E-mail: bevilacqua@ coc.ufrj.br http://orcid.org/0000-0002-7695-1385

${ }^{4}$ DEMEC, IPRJ/UERJ, Nova Friburgo, RJ, Brasil - E-mail: gmarinho@iprj.uerj.br http://orcid.org/0000-0003-26814398

5 DEMEC, IPRJ/UERJ, Nova Friburgo, RJ, Brasil - E-mail: diegoknupp@iprj.uerj.br https://orcid.org/0000-0001-95345623

${ }^{6}$ DEMEC, IPRJ/UERJ, Nova Friburgo, RJ, Brasil - E-mail: jflavio@iprj.uerj.br https://orcid.org/0000-0002-0219-3198

${ }^{7}$ DEMEC, IPRJ/UERJ, Nova Friburgo, RJ, Brasil - E-mail: ajsneto@iprj.uerj.br http://orcid.org/0000-0002-9616-6093
} 
tivo sob condições termodinâmicas estáveis. Baseado nestas e outras considerações, estes autores propuseram a seguinte equação para descrever tal fenômeno em um domínio espacial unidimensional:

$$
\frac{\partial \phi}{\partial t}=\beta K_{2} \frac{\partial^{2} \phi}{\partial x^{2}}-\beta(1-\beta) K_{4} \frac{\partial^{4} \phi}{\partial x^{4}}
$$

em que $\phi$ é a concentração, $x$ a coordenada espacial e $t$ o tempo. Nesta nova abordagem, o processo de difusão está associado a dois fluxos, um correspondente à fração $\beta$, fluxo primário sujeito à difusão clássica, e outro à fração $(1-\beta)$, fluxo secundário. Os coeficientes $K_{2}$ e $K_{4}$ estão relacionados respectivamente aos fluxos primário e secundário. Para $\beta=1$, a Eq. (1.1) volta a ser a equação de difusão clássica de Fick.

O efeito de fluxo bimodal pode ser observado nesta equação através da presença do termo diferencial de quarta ordem. Não cabe neste trabalho detalhar a dedução desta equação, para tanto, maiores detalhes podem ser obtidos através da leitura do trabalho de Bevilacqua e coautores [1]. Por seu aspecto inovador, Eq. (1.1) tem sido investigada por diversos autores. Citamos o trabalho de Silva Neto e colaboradores [11] que resolveram numericamente a Eq. (1.1) e realizaram o estudo de sensibilidade visando a estimativa dos parâmetros. Em artigos posteriores, a mesma equipe de pesquisadores apresentou soluções para a estimativa dos parâmetros $K_{2}$ e $K_{4}$ da Eq. (1.1) utilizando como metodologias a inferência Bayesiana e a máxima verossimilhança $[4,12]$.

Vasconcellos et al. [14] desenvolveram uma solução baseada no Método de Volumes Finitos para a versão em regime permanente da Eq. (1.1). Este trabalho dedicou-se particular atenção para as dificuldades numéricas relacionadas às condições de contorno (Dirichlet, Neumann e Robin) e das aproximações do termo de fluxo secundário.

No presente trabalho, visando ampliar o uso da formulação de fluxo bimodal, incluiu-se um termo advectivo, resultando na seguinte equação:

$$
\frac{\partial \phi}{\partial t}+v \frac{\partial \phi}{\partial x}=\beta K_{2} \frac{\partial^{2} \phi}{\partial x^{2}}-\beta(1-\beta) K_{4} \frac{\partial^{4} \phi}{\partial x^{4}}
$$

em que $v$ é a velocidade na direção $x$.

Serão apresentados alguns resultados para a solução numérica da Eq. (1.2) empregando o Método de Diferenças Finitas.

\section{MODELO NUMÉRICO}

O Método de Diferenças Finitas [6] é, provavelmente, o método numérico mais empregado para resolver problemas de difusão de Fick e, portanto, as técnicas numéricas utilizadas na discretização desta equação já estão há muito consagradas.

Contudo, o mesmo não se pode afirmar quando se inclui o termo com a derivada de quarta ordem. Equações contendo derivadas desta ordem não são comumente encontradas em modelos envolvendo fenômenos de transporte e, portanto, são raros os trabalhos numéricos disponíveis na literatura que lidam com tais equações. 
Uma dificuldade, que foi investigada por Vasconcellos et al. [14] para o Método de Volumes Finitos, está relacionada com a aproximação da derivada de segunda ordem. Tradicionalmente a opção escolhida para discretizar este termo é a seguinte:

$$
\left.\frac{\partial^{2} \phi}{\partial x^{2}}\right|_{x_{i}}=\frac{\phi\left(x_{i}-h\right)-2 \phi\left(x_{i}\right)+\phi\left(x_{i}+h\right)}{h^{2}}+\left.\frac{1}{12} \frac{\partial^{4} \phi}{\partial x^{4}}\right|_{x_{i}} h^{2}+\cdots
$$

em que $x_{i}$ é a coordenada do $i$-ésimo nó e $h$ é o espaçamento adotado em uma malha com os nós igualmente espaçados.

Um aspecto que pode passar desapercebido por quem modela esta equação é que a aproximação usual para o termo de segunda ordem desconsidera os termos de derivada quarta ou superior. Isto significa que ao se modelar o termo de segunda ordem da Eq. (1.2) com a aproximação definida pela Eq. (2.1) se está desconsiderando derivadas que são da mesma ordem do termo responsável pela difusão secundária. Dependendo da relação $K_{2} / K_{4}$ erros numéricos significativos poderão ocorrer.

Por esta razão, ao modelar o termo de segunda ordem neste trabalho empregou-se a seguinte aproximação:

$$
\begin{aligned}
\left.\frac{\partial^{2} \phi}{\partial x^{2}}\right|_{x_{i}} \approx & \frac{-\phi\left(x_{i}-2 h\right)+16 \phi\left(x_{i}-h\right)-30 \phi\left(x_{i}\right)}{12 h^{2}} \\
& +\frac{16 \phi\left(x_{i}+h\right)-\phi\left(x_{i}+2 h\right)}{12 h^{2}}+O\left(h^{4}\right)
\end{aligned}
$$

Apesar desta aproximação utilizar cinco nós, dois a mais do que é usualmente adotado neste tipo de aproximação, isto não irá comprometer a solução do sistema de equações lineares, visto que a aproximação para o termo de difusão secundária também conterá cinco nós.

Os demais termos da Eq. (1.2) são definidos da seguinte maneira:

$$
\begin{gathered}
\left.\frac{\partial \phi}{\partial t}\right|_{x_{i}, t+\Delta t} \approx \frac{\phi^{t+\Delta t}\left(x_{i}\right)-\phi^{t}\left(x_{i}\right)}{\Delta t}+O(\Delta t) \\
\left.\frac{\partial \phi}{\partial x}\right|_{x_{i}, t+\Delta t} \approx \frac{\phi^{\theta}\left(x_{i}-2 h\right)-8 \phi^{\theta}\left(x_{i}-h\right)}{12 h}+\frac{8 \phi^{\theta}\left(x_{i}+h\right)-\phi^{\theta}\left(x_{i}+2 h\right)}{12 h}+O\left(h^{4}\right) \\
\left.\frac{\partial^{4} \phi}{\partial x^{4}}\right|_{x_{i}, t+\Delta t} \approx \frac{\phi^{\theta}\left(x_{i}-2 h\right)-4 \phi^{\theta}\left(x_{i}-h\right)+6 \phi^{\theta}\left(x_{i}\right)}{h^{4}}+ \\
\frac{-4 \phi^{\theta}\left(x_{i}+h\right)+\phi^{\theta}\left(x_{i}+2 h\right)}{h^{4}}+O\left(h^{4}\right)
\end{gathered}
$$

Na Eq. (2.4) a derivada de quinta ordem é a derivada de menor ordem desconsiderada e na Eq. (2.5) é uma derivada de sexta ordem. Desta forma há a garantia que nenhuma derivada de quarta ordem foi desconsiderada na série de Taylor, o que poderia influenciar na solução numérica, dependendo dos valores de $K_{2} / K_{4}$. 
Estas aproximações não poderão ser utilizadas para o primeiro e segundo nó da malha e também para o último e penúltimo nó da malha. Para estes quatro nós ficam reservadas equações especiais que envolverão as condições de contorno do problema e que serão detalhadas na seção 3.

A Eq. (1.2), em sua forma discretizada, é definida como:

$$
\begin{aligned}
& \left(\frac{h}{\Delta t}\right) \phi_{i}^{t+\Delta t}=\left(-\frac{v}{12 h}-\frac{\Gamma_{2}}{12}-\Gamma_{4}\right) \phi_{i-2}^{\theta}+\left(\frac{2 v}{3 h}+\frac{4 \Gamma_{2}}{3}+4 \Gamma_{4}\right) \phi_{i-1}^{\theta} \\
& \left(-\frac{2 v}{3 h}+\frac{4 \Gamma_{2}}{3}+4 \Gamma_{4}\right) \phi_{i+1}^{\theta}+\left(\frac{v}{12 h}-\frac{\Gamma_{2}}{12}-\Gamma_{4}\right) \phi_{i+2}^{\theta}+ \\
& \left(-\frac{5 \Gamma_{2}}{2}-6 \Gamma_{4}\right) \phi_{i}^{\theta}+\left(\frac{h}{\Delta t}\right) \phi_{i}^{t}
\end{aligned}
$$

em que $\Gamma_{2}=\beta K_{2} / h^{2}$ e $\Gamma_{4}=\beta(1-\beta) K_{4} / h^{4}$.

Neste momento deve-se escolher o valor de $\theta$, isto é, qual a interpolação no tempo que será adotada. Uma opção seria fazer $\phi^{\theta}=\phi^{t}$, ou seja, escolher uma formulação totalmente explícita [7]. Por este caminho evita-se resolver um sistema linear de equações, o que é algo interessante, porém há a conhecida restrição de estabilidade visto que esta formulação é condicionalmente estável. O valor de $\Delta t$ que satisfaz a condição de estabilidade para a Eq. (2.6) é ainda mais proibitivo do que no problema clássico de difusão. Isto faz com que a opção de usar uma formulação totalmente explícita deva ser descartada.

Como outras opções de formulação explícita, como $\phi^{\theta}=1 / 2\left(\phi^{t+\Delta t}+\phi^{t}\right)$ (Crank-Nicolson), necessitarão também resolver um sistema de equações lineares, optou-se por utilizar a formulação implícita, isto é, $\phi^{\theta}=\phi^{t+\Delta t}$, que mesmo com a presença do termo de difusão secundária continuará sendo incondicionalmente estável. Sendo assim, a Eq. (2.6) é reescrita:

$$
\begin{aligned}
& \left(\frac{h}{\Delta t}+\frac{5 \Gamma_{2}}{2}+6 \Gamma_{4}\right) \phi_{i}^{t+\Delta t}=\left(-\frac{v}{12 h}-\frac{\Gamma_{2}}{12}-\Gamma_{4}\right) \phi_{i-2}^{t+\Delta t}+ \\
& \left(\frac{2 v}{3 h}+\frac{4 \Gamma_{2}}{3}+4 \Gamma_{4}\right) \phi_{i-1}^{t+\Delta t}+\left(-\frac{2 v}{3 h}+\frac{4 \Gamma_{2}}{3}+4 \Gamma_{4}\right) \phi_{i+1}^{t+\Delta t}+ \\
& \left(\frac{v}{12 h}-\frac{\Gamma_{2}}{12}-\Gamma_{4}\right) \phi_{i+2}^{t+\Delta t}+\left(\frac{h}{\Delta t}\right) \phi_{i}^{t}
\end{aligned}
$$

A Eq. (2.7) representa a equação para todos os nós do problema, com exceção dos dois primeiros e dos dois últimos.

\section{CONDIÇÕES DE CONTORNO}

Para a equação de difusão bimodal é necessário definir quatro condições de contorno, visto que há um termo contendo uma diferencial de quarta ordem. Para definir a primeira condição de contorno em $x=0$ será utilizada a seguinte equação:

$$
w_{10} \phi(x=0, t+\Delta t)+\left.w_{11} \frac{\partial \phi}{\partial x}\right|_{x=0, t+\Delta t}+\left.w_{12} \frac{\partial^{2} \phi}{\partial x^{2}}\right|_{x=0, t+\Delta t}=f_{1}(x=0, t+\Delta t)
$$


em que $w_{10}, w_{11}, w_{12}$ são valores conhecidos. Por exemplo, se a primeira condição de contorno em $x=0$ for $\phi(x)=2$ (Dirichlet) então $w_{10}=1, w_{11}=0, w_{12}=0$ e $f_{1}(x=0, t+\Delta t)=2$.

Fazendo uma expansão em série de Taylor, considerando os cinco primeiros nós do domínio de cálculo chega-se a equação do primeiro nó do domínio em função de $w_{10}, w_{11}, w_{12}$ e $f_{1}(x=$ $0, t+\Delta t)$. Esta equação fica:

$$
\begin{aligned}
& \left(w_{10}-\frac{25}{12 h} w_{11}+\frac{35}{12 h^{2}} w_{12}\right) \phi_{1}^{t+\Delta t}=\left(-\frac{4}{h} w_{11}+\frac{26}{3 h^{2}} w_{12}\right) \phi_{2}^{t+\Delta t}+ \\
& \left(\frac{3}{h} w_{11}-\frac{19}{2 h^{2}} w_{12}\right) \phi_{3}^{t+\Delta t}+\left(-\frac{4}{3 h} w_{11}+\frac{14}{3 h^{2}} w_{12}\right) \phi_{4}^{t+\Delta t}+ \\
& \left(\frac{1}{4 h} w_{11}-\frac{11}{12 h^{2}} w_{12}\right) \phi_{5}^{t+\Delta t}+f_{1}(x=0, t+\Delta t)
\end{aligned}
$$

A segunda condição de contorno em $x=0$ é definida de forma similar ao que foi feito para a primeira condição de contorno. Sendo assim tem-se a seguinte equação para a condição de contorno:

$$
w_{20} \phi(x=0, t+\Delta t)+\left.w_{21} \frac{\partial \phi}{\partial x}\right|_{x=0, t+\Delta t}+\left.w_{22} \frac{\partial^{2} \phi}{\partial x^{2}}\right|_{x=0, t+\Delta t}=f_{2}(x=0, t+\Delta t)
$$

em que $w_{20}, w_{21}, w_{22}$ são valores conhecidos. Novamente, utilizando uma expansão em série de Taylor, considerando os cinco primeiros nós do domínio de cálculo, chega-se a equação do segundo nó do domínio em função de $w_{20}, w_{21}, w_{22}$ e $f_{2}(x=0, t+\Delta t)$. Esta equação é definida como:

$$
\begin{aligned}
& \left(\frac{4}{h} w_{21}-\frac{26}{3 h^{2}} w_{22}\right) \phi_{2}^{t+\Delta t}=\left(-w_{20}+\frac{25}{12 h} w_{21}-\frac{35}{12 h^{2}} w_{22}\right) \phi_{1}^{t+\Delta t}+ \\
& \left(\frac{3}{h} w_{21}-\frac{19}{2 h^{2}} w_{22}\right) \phi_{3}^{t+\Delta t}+\left(-\frac{4}{3 h} w_{21}+\frac{14}{3 h^{2}} w_{22}\right) \phi_{4}^{t+\Delta t}+ \\
& \left(\frac{1}{4 h} w_{21}-\frac{11}{12 h^{2}} w_{22}\right) \phi_{5}^{t+\Delta t}+f_{2}(x=0, t+\Delta t)
\end{aligned}
$$

O procedimento para informar as condições de contorno em $x=L$ é análogo ao adotado para informar as condições de contorno em $x=0$. Sendo assim, serão utilizadas as seguintes equações:

$$
e_{10} \phi(x=L, t+\Delta t)+\left.e_{11} \frac{\partial \phi}{\partial x}\right|_{x=L, t+\Delta t}+\left.e_{12} \frac{\partial^{2} \phi}{\partial x^{2}}\right|_{x=L, t+\Delta t}=f_{3}(x=L, t+\Delta t)
$$

em que $L$ é o comprimento do domínio de cálculo e $e_{10}, e_{11}, e_{12}, e_{20}, e_{21}, e_{22}$ são valores conhecidos. Repete-se o mesmo procedimento para se obter a equação discretizada a partir de expansões em série de Taylor. Neste caso, a equação é definida como:

$$
\begin{aligned}
& \left(e_{10}+\frac{25}{12 h} e_{11}+\frac{35}{12 h^{2}} e_{12}\right) \phi_{N}^{t+\Delta t}=\left(\frac{4}{h} e_{11}+\frac{26}{3 h^{2}} w_{12}\right) \phi_{N-1}^{t+\Delta t}+ \\
& \left(-\frac{3}{h} e_{11}-\frac{19}{2 h^{2}} e_{12}\right) \phi_{N-2}^{t+\Delta t}+\left(\frac{4}{3 h} e_{11}+\frac{14}{3 h^{2}} e_{12}\right) \phi_{N-3}^{t+\Delta t}+ \\
& \left(-\frac{1}{4 h} e_{11}-\frac{11}{12 h^{2}} e_{12}\right) \phi_{N-4}^{t+\Delta t}+f_{3}(x=L, t+\Delta t)
\end{aligned}
$$


A segunda condição de contorno em $x=L$ é definida de forma idêntica ao que foi realizado para a primeira condição de contorno. Sendo assim, tem-se a seguinte equação para a condição de contorno:

$$
e_{20} \phi(x=L, t+\Delta t)+\left.e_{21} \frac{\partial \phi}{\partial x}\right|_{x=L, t+\Delta t}+\left.e_{22} \frac{\partial^{2} \phi}{\partial x^{2}}\right|_{x=0, t+\Delta t}=f_{4}(x=0, t)
$$

em que $e_{20}, e_{21}, e_{22}$ também são valores conhecidos. Fazendo uma expansão em série de Taylor, considerando os cinco últimos nós do domínio de cálculo chega-se a equação do penúltimo nó do domínio em função de $e_{20}, e_{21}, e_{22}$ e $f_{4}(x=L, t+\Delta t)$. Dessa forma, obtém-se a equação a seguir:

$$
\begin{aligned}
& \left(-\frac{4}{h} e_{21}-\frac{26}{3 h^{2}} e_{22}\right) \phi_{N-1}^{t+\Delta t}=\left(-e_{20}-\frac{25}{12 h} e_{21}-\frac{35}{12 h^{2}} e_{22}\right) \phi_{N}^{t+\Delta t}+ \\
& \left(-\frac{3}{h} e_{21}-\frac{19}{2 h^{2}} e_{22}\right) \phi_{N-2}^{t+\Delta t}+\left(\frac{4}{3 h} e_{21}+\frac{14}{3 h^{2}} e_{22}\right) \phi_{N-3}^{t+\Delta t}+ \\
& \left(-\frac{1}{4 h} e_{21}-\frac{11}{12 h^{2}} e_{22}\right) \phi_{N-4}^{t+\Delta t}+f_{4}(x=L, t+\Delta t)
\end{aligned}
$$

Vale ressaltar que estas quatro equações satisfazem tão somente as condições de contorno, e não satisfazem a equação diferencial.

\section{RESULTADOS E DISCUSSÃO}

Os resultados apresentados nesta seção referem-se a dois problemas com condições iniciais e de contorno descritas na Tabela 1. Os problemas analisados aqui correspondem a situações idênticas àquelas tratadas por Silva et al. [11]. Em todos os casos apresentados tem-se $L=1.00, \beta=0.20 \mathrm{e}$ $K_{2}=1 \times 10^{-3}$. Os valores de $K_{4}$ e $v$ variaram de acordo com o propósito do problema analisado.

$\mathrm{O}$ primeiro resultado apresentado, em que $K_{4}=v=0$, visto na Figura 1, apresenta a norma da diferença entre a solução numérica e analítica, que está definida a seguir:

$$
\phi_{\text {analítico }}(x, t)=e^{-\beta K_{2} \pi^{2} t} e^{-\beta(1-\beta) K_{4} \pi^{4} t} \sin (\pi x)
$$

Esta solução analítica é válida para o problema definido na Tabela 1 como problema A e considerando $v=0$ e $K_{4}=0$. Como pode ser observado, o padrão da redução do erro com o refino da malha é exatamente como o esperado. Outras comparações com soluções analíticas foram realizadas e não serão apresentadas aqui. Mas este conjunto de comparações assegura que a metodologia numérica está corretamente implementada em um código em desenvolvimento.

Na Figura 2 são apresentadas $\phi(x, t)$ analítico e numérico para o problema definido na Tabela 1 como problema A e especificando $K_{4}=v=0$. Como a solução numérica e analítica são bastante próximas, não se consegue distinguir uma da outra. Nestas as simulações utilizou-se $\Delta t=1[s] \mathrm{e}$ $h=0.01[m]$.

O próximo resultado, na Figura 3, mostra a comparação entre a solução do problema definido na Tabela 1 como problema B, considerando $v=0.1[\mathrm{~m} / \mathrm{s}]$ e $K_{4}=0$, obtida a partir da metodologia apresentada aqui, e a solução utilizando a rotina NDSolve do Mathematica, com controle 
Tabela 1: Problemas estudados.

\section{Problema A Problema B}

$$
\text { Condição Inicial } \quad \phi(x, 0)=\sin \left(\frac{x}{L} \pi\right) \quad \phi(x, 0)=1+\sin ^{100}\left(\frac{x}{L} \pi\right)
$$

$\begin{array}{lll}\text { Contorno à esquerda } 1 \quad \phi(0, t)=0 & \phi(0, t)=1\end{array}$

Contorno à esquerda $\left.2 \quad \frac{\partial^{2} \phi}{x^{2}}\right|_{x=0}=\left.0 \quad \frac{\partial \phi}{x}\right|_{x=0}=0$

Contorno à direita $1 \quad \phi(L, t)=0 \quad \phi(L, t)=1$

Contorno à direita $\left.2 \quad \frac{\partial^{2} \phi}{x^{2}}\right|_{x=L}=\left.0 \quad \frac{\partial \phi}{x}\right|_{x=L}=0$

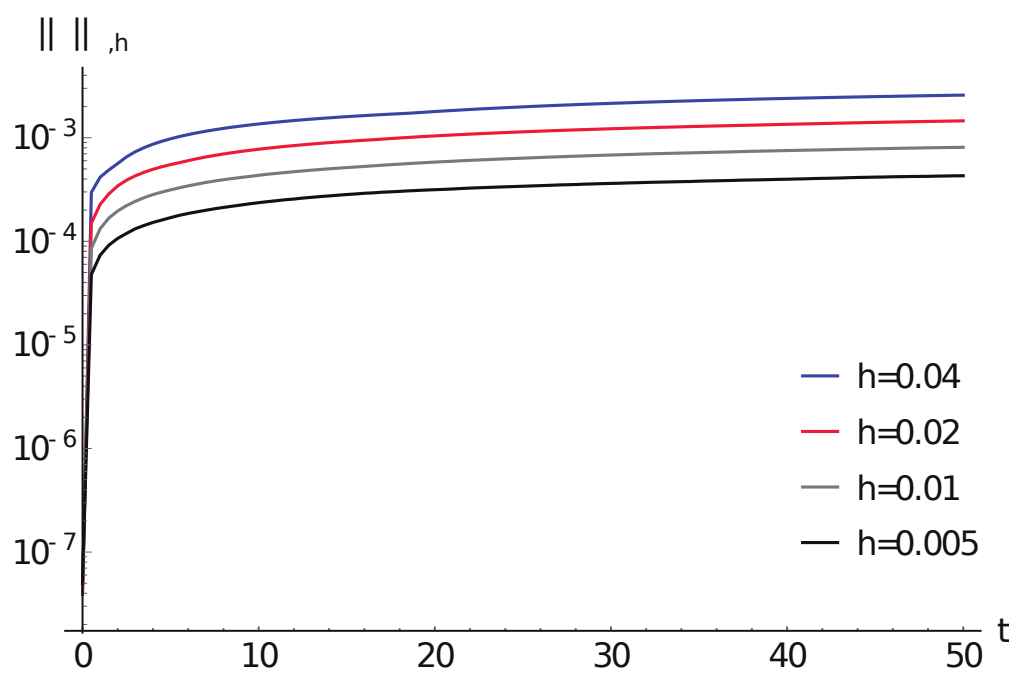

Figura 1: Comparação com a solução analítica.

automático de erro relativo e absoluto, para solução numérica de equações diferenciais. Nestas as simulações utilizou-se $\Delta t=0.01[\mathrm{~s}]$ e $h=0.01[\mathrm{~m}]$. Neste caso, o efeito do fluxo secundário foi desconsiderado mas o terma advectivo não. Olhando-se atentamente a figura pode-se ver uma discreta diferença nos dois últimos pontos de máximo da função. Contudo, como esta diferença 


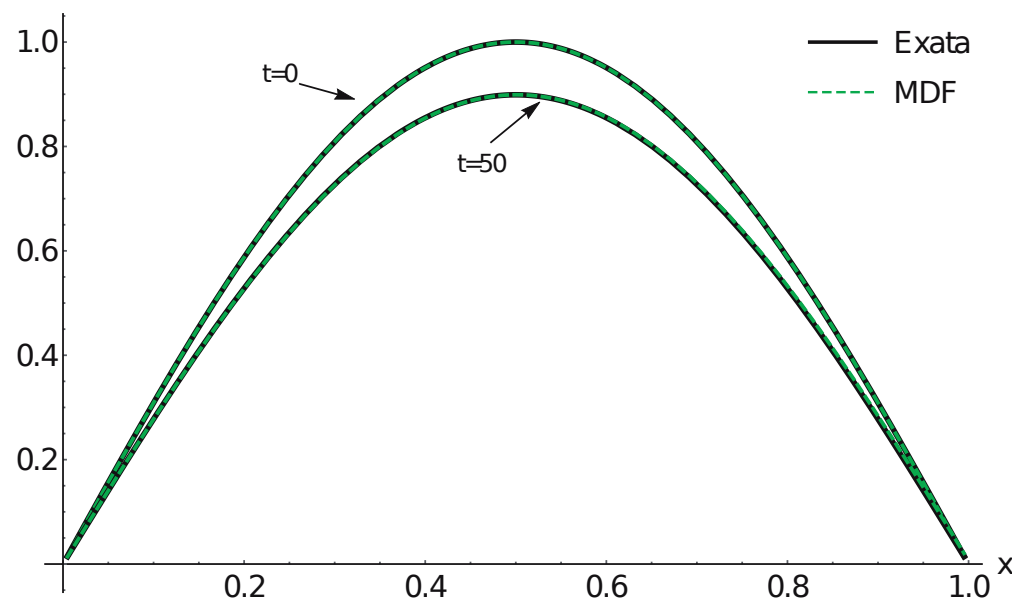

Figura 2: Comparação com a solução analítica.

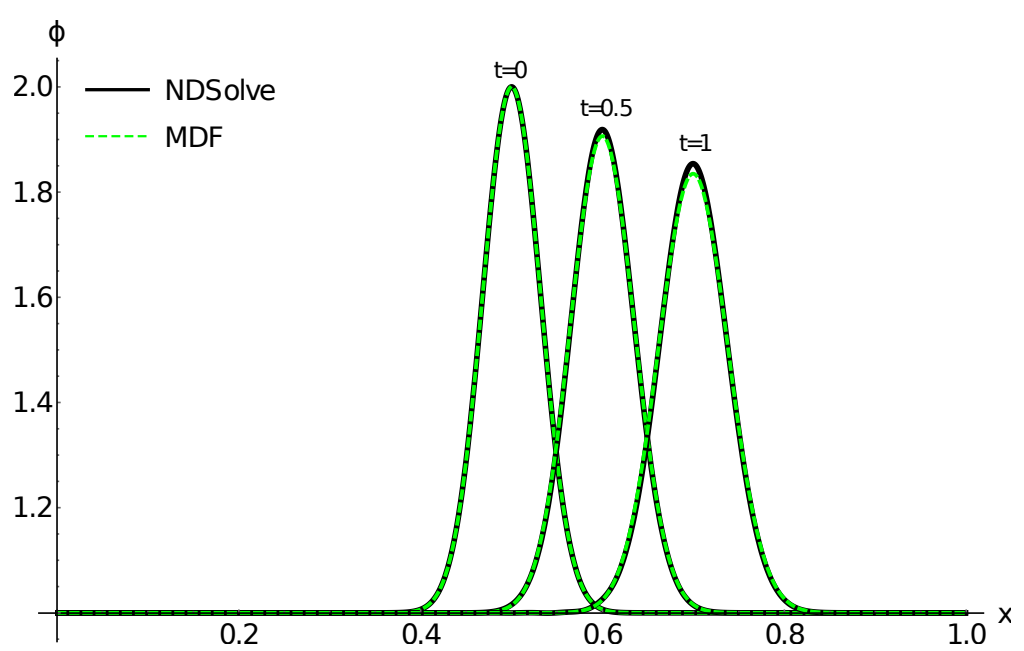

Figura 3: Comparação com a solução empregando o NDSolve.

não é significativa pode-se considerar que a implementação do termo de advecção do código foi realizada com sucesso.

Na Figura 4 estão apresentados os resultados considerando três valores distintos de $K_{4}$, considerando as condições iniciais e de contorno definidas para o problema B e uma velocidade $v=0.2[\mathrm{~m} / \mathrm{s}]$ no tempo $t=0.5 \mathrm{~s}$. O efeito da difusão secundária fica bem visível nesta figura. Pode-se observar claramente valores de $\phi<1$ quando $K_{4} \neq 0$.

Pode-se perceber que o valor máximo de cada uma das curvas, apesar de serem diferentes, ocorrem no mesmo ponto, neste caso numa posição próxima a $0.6[\mathrm{~m}]$. Pode-se concluir que a 


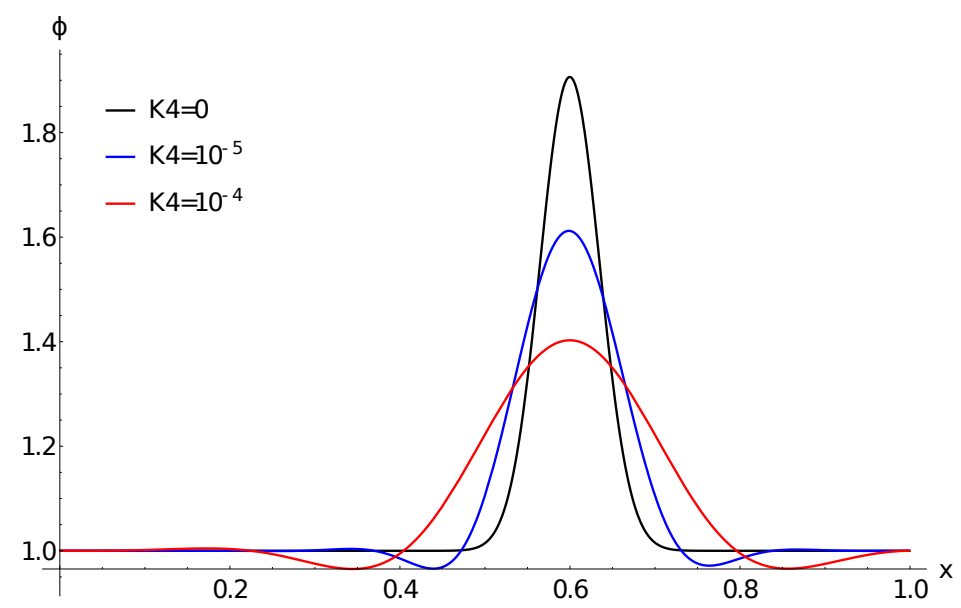

Figura 4: Solução para o caso 4.

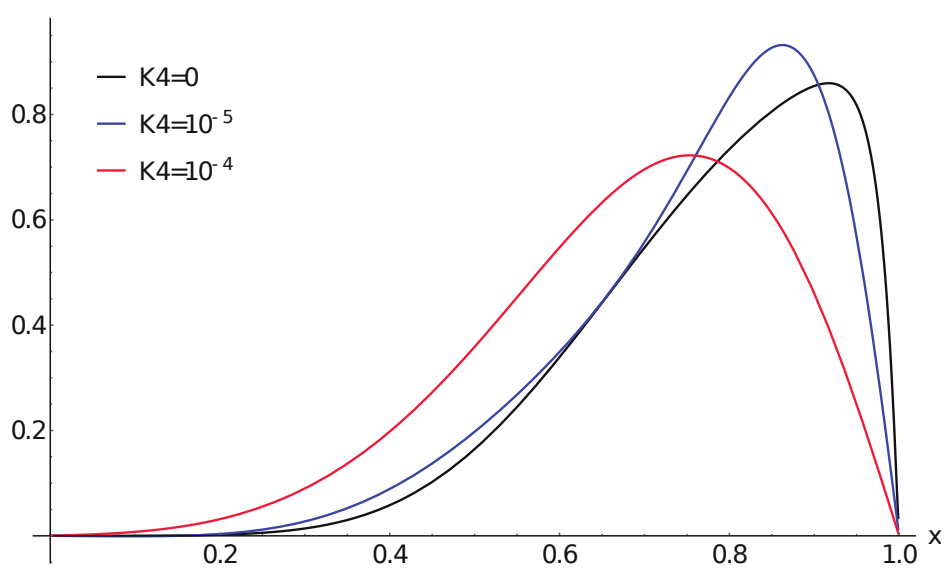

Figura 5: Solução com advecção dominante.

presença do termo de fluxo secundário não retarda o avanço da frente de difusão. Esta conclusão se mostrou estar errada, como será demonstrado com os próximos resultados.

Na Figura 5 apresentamos um resultado onde $t=50 \mathrm{~s}, v=0.01[\mathrm{~m} / \mathrm{s}]$ e $K_{4}$ é variável. Neste caso, o resultado deixou de ter uma simetria. A consideração feita anteriormente de que a presença da difusão secundária não altera o avanço da frente de difusão se mostrou ser precipitada. Claramente a presença do termo de difusão secundária retarda o avanço da frente de difusão e altera os valores máximos desta frente. 


\title{
5 CONCLUSÕES E TRABALHOS FUTUROS
}

Neste trabalho incluiu-se o termo de advecção na equação da difusão bimodal proposta por Bevilacqua et al. [1]. A equação diferencial resultante foi discretizada utilizando o Método de Diferenças Finitas e resultados numéricos foram apresentados.

O resultado mais importante vem da Figura 5. Ele demonstra que a difusão secundária altera o comportamento de um escoamento com difusão dominante. Outros modelos de difusão anômala já haviam previsto isto, mas para este tipo de modelo é a primeira vez que isto é apresentado.

As soluções numéricas obtidas para os casos considerados permitiram avaliar separadamente o efeito advectivo, da difusão primária e da secundária em diferentes condições iniciais e de contorno.

Com a solução desenvolvida, será possível calibrar o novo modelo de advecção e difusão bimodal com dados experimentais para uso em aplicações onde ocorram efeitos de retenção. Com tal objetivo, a solução será também implementada para duas dimensões, bem como será realizado o estudo de sensibilidade e finalmente serão aplicadas técnicas para solução de problemas inversos.

Para a solução de problemas inversos, busca-se com frequência soluções para os problemas diretos correspondentes que sejam acurados e obtidas com tempo computacional reduzido. A solução aqui apresentada atende estes requisitos.

\section{AGRADECIMENTOS}

Os autores agradecem o apoio financeiro fornecido da FAPERJ, Fundação Carlos Chagas Filho de Amparo à Pesquisa do Estado do Rio de Janeiro, do CNPq, Conselho Nacional de Desenvolvimento Científico e Tecnológico, e da CAPES, Fundação Coordenação de Aperfeiçoamento de Pessoal de Nivel Superior.

\begin{abstract}
This paper presents a methodology for the solution of a bimodal advection and diffusion problem using the Finite Differences Method. In addition to the advective transport term and primary diffusion (which corresponds to the Fick's flux), the bimodal diffusion equation includes a term relative to a secondary flow that is modeled by a fourth order differential term. The problem was analyzed for different initial and boundary conditions and the results are compatible with those presented in previous studies in the literature.
\end{abstract}

Keywords: bimodal diffusion, anomalous diffusion, Finite Difference Method, fourthorder differential equation.

\section{REFERÊNCIAS}

[1] L. Bevilacqua, A.C.N.R. Galeão \& F.P. Costa. A new analytical formulation of retention effects on particle diffusion processes. Anais da Academia Brasileira de Ciências, 83 (2011), 1443-1464. URL http://www.scielo.br/scielo.php?script=sci_arttext\&pid= S0001-37652011000400031\&nrm=iso. 
[2] L. Bevilacqua, A.C.N.R. Galeão \& F.P. Costa. On the significance of higher order differential terms in diffusion processes. Journal of the Brazilian Society of Mechanical Sciences and Engineering, 33 (2011), 166-175. URL http://www.scielo.br/scielo.php?script=sci_arttext\&pid= S0001-37652011000400031\&nrm=iso.

[3] L. Bevilacqua, A.C.N.R. Galeão, J.G. Simas \& A.P.R. Doce. A new theory for anomalous diffusion with a bimodal flux distribution. Journal of the Brazilian Society of Mechanical Sciences and Engineering, (2013), 1-10. doi:10.1007/s40430-013-0041-y. URL http://dx. doi .org/10 . 1007/ s40430-013-0041-y.

[4] Diego Campos Knupp, Luciano Gonçalves Silva, Luiz Bevilacqua, Augusto César Noronha Rodrigues Galeão \& Antônio José da Silva Neto. Inverse analysis of a new anomalous diffusion model employing maximum likelihood and Bayesian estimation. In Antônio José da Silva Neto, Oreste Llanes Santiago \& G.N. Silva (editors), "Mathematical Modeling and Computational Intelligence in Engineering Applications", volume 36. Springer International Publishing, Cham (2016), chapter 7, pp. 89-104. doi:10.1007/978-3-319-38869-4\{\_\}7. URL http://cascavel . ufsm. br/revistas/ ojs-2.2.2/index.php/cienciaenatura/article/view/13184.

[5] V. Ganti, M.M. Meerschaert, E. Foufoula-Georgiou, E. Viparelli \& G. Parker. Normal and anomalous diffusion of gravel tracer particles in rivers. Journal of Geophysical Research: Earth Surface, 115(F2) (2010). doi:10.1029/2008JF001222. URL http://doi . wiley . com/10.1029/2008JF001222.

[6] R.J. LeVeque. "Finite Difference Methods for Ordinary and Partial Differential Equations: Steadystate and Time-dependent Problems". SIAM, Philadelphia (2007), 339 pp.

[7] C.R. Maliska. "Transferência de calor e mecânica dos fluidos computacional”. LTC Editora (1994), $472 \mathrm{pp}$.

[8] P.F. Nealey, R.E. Cohen \& A.S. Argon. Limited-supply non-Fickian diffusion in glassy polymers. Polymer, 36(19) (1995), 3687-3695. doi:10.1016/0032-3861(95)93771-D. URL http://www . sciencedirect.com/science/article/pii/003238619593771D.

[9] J.I. Ramos. On the numerical treatment of an ordinary differential equation arising in one-dimensional non-Fickian diffusion problems. Computer Physics Communications, 170(3) (2005), 231238. doi:10.1016/j.cpc.2005.04.004. URL http://www. sciencedirect. com/science/article/ $\mathrm{pii} / \mathrm{S} 0010465505002687$.

[10] M.J. Saxton. Anomalous diffusion due to obstacles: a Monte Carlo study. Biophysical Journal, 66(2, Part 1) (1994), 394-401. doi:http://dx.doi.org/10.1016/S0006-3495(94)80789-1. URL http://www . sciencedirect.com/science/article/pii/S0006349594807891.

[11] L.G. Silva, D.C. Knupp, L. Bevilacqua, A.C.N.R. Galeão \& A.J.S. Neto. Inverse problem in anomalous diffusion with uncertainty propagation. Computer Assisted Methods in Engineering and Science; Vol 21 No 3/4 (2014), (2017). URL http://cames.ippt.gov.pl/index.php/cames/article/ view/43.

[12] L.G.d. Silva, D.C. Knupp, L. Bevilacqua, A.C.N.R. Galeão \& A.J.D. Silva Neto. Formulação e solução de um problema inverso de difusão anômala com técnicas estocástica. Ciência e Natura, 36(3) (2014). doi:10.5902/2179460X13184. URL http://cascavel.ufsm.br/revistas/ojs-2.2.2/index. php/cienciaenatura/article/view/13184. 
[13] P.M.M. Smith \& M.M.M. Fisher. Non-Fickian diffusion of water in melamine-formaldehyde resins. Polymer, 25(1) (1984), 84-90. doi:10.1016/0032-3861(84)90271-4. URL http://linkinghub. elsevier.com/retrieve/pii/0032386184902714.

[14] J. Vasconcellos, G. Marinho \& J. Zani. Análise numérica da equação da difusão anômala com fluxo bimodal. Revista Internacional de Métodos Numéricos para Cálculo y Diseño en Ingeniería, 33(3-4) (2017), 242-249. doi:10.1016/j.rimni.2016.05.001. URL https://www.scipedia.com/public/ Vasconcellos_et_al_2016a.

[15] J. Wu \& K.M. Berland. Propagators and Time-Dependent Diffusion Coefficients for Anomalous Diffusion. Biophysical Journal, 95(4) (2008), 2049-2052. doi:http://dx.doi.org/10. 1529/biophysj.107.121608. URL http://www.sciencedirect.com/science/article/pii/ S0006349508701620. 